\title{
BMJ Open An exploratory spatial analysis of overweight and obesity among children and adolescents in Shandong, China
}

\author{
Wenzhe Qin, ${ }^{1}$ Lu Wang, ${ }^{2}$ Lingzhong Xu, ${ }^{1}$ Long Sun, ${ }^{\oplus 1}$ Jiajia Li, ${ }^{1}$ Jiao Zhang, ${ }^{1}$ \\ Hui Shao ${ }^{3}$
}

To cite: Qin W, Wang L, $\mathrm{Xu} \mathrm{L}$, et al. An exploratory spatial analysis of overweight and obesity among children and adolescents in Shandong, China. BMJ Open 2019;9:e028152. doi:10.1136/ bmjopen-2018-028152

- Prepublication history for this paper is available online. To view these files, please visit the journal online (http://dx.doi. org/10.1136/bmjopen-2018028152).

WQ and LW contributed equally.

Received 26 November 2018 Revised 13 June 2019 Accepted 09 July 2019

Check for updates

(c) Author(s) (or their employer(s)) 2019. Re-use permitted under CC BY-NC. No commercial re-use. See rights and permissions. Published by BMJ.

${ }^{1}$ School of Public Health, Shandong University, Jinan, China

${ }^{2}$ Shandong Provincial Medical Examination Office, Shandong Province Health and Family Planning Commission, Jinan, China

${ }^{3}$ Department of Global Health Systems and Development School of Public Health and Tropical Medicine, Tulane University, New Orleans, Louisiana, USA

Correspondence to Dr Lingzhong Xu; Izxu@sdu.edu.cn

\section{ABSTRACT}

Objective Identifying the spatial patterns of childhood overweight/obesity (OW/OB) can help to guide resource allocation for preventive intervention in China. This study aims to estimate rates of childhood OW/OB across counties within Shandong Province, using geographic techniques to identify sex-specific spatial patterns of childhood OW/OB as well as the presence of spatial clusters.

Design Cross-sectional study.

Setting Shandong Province in China.

Participants and methods Data on 6216076 children and adolescents aged 7-18 years from the Primary and Secondary Schoolchildren Physical Examination Database for Shandong Province were used in this study. Spatial patterns of sex-specific prevalence of childhood OW/OB were mapped. Global autocorrelation statistic (Moran's I) and the Local Indicator of Spatial Association (LISA) were applied to assess the degree of spatial autocorrelation.

Results The overall prevalence of childhood OW/OB in Shandong province were $15.05 \%$ and $9.23 \%$, respectively. Maps of the sex-specific prevalence of OW/OB demonstrate a marked geographical variation of childhood OW/OB in different regions. Prevalence of childhood OW/ OB had a significant positive spatial autocorrelation among both boys and girls. LISA analysis identified significant clusters (or 'hot spots') of childhood OW/OB in the eastern coastal region, central region and southwestern region. Conclusions The prevalence of childhood OW/OB is highly spatially clustered. Geographically focused appropriate intervention should be introduced in current childhood OW/OB prevention and control strategy.

\section{INTRODUCTION}

Childhood obesity is one of the most serious public health challenges in the 21st century. ${ }^{1}$ The prevalence of overweight and obesity (OW/OB) among children and adolescent has been rising rapidly in many developed countries and is affecting many low and middle-income countries. ${ }^{2-5}$ With rapid economic development over the past decades, China has also witnessed an increase in the prevalence of childhood obesity. ${ }^{67}$ In 2013, approximately $23.0 \%$ and $14.0 \%$ of Chinese boys and girls aged 2-19 years were

\section{Strengths and limitations of this study}

- With a large sample size, this study is a secondary analysis of census database of primary and secondary schoolchildren aged 7-18 years old in publicly maintained schools in Shandong Province.

- Geographic techniques to identify sex-specific spatial patterns of childhood overweight/obesity (OW/ $\mathrm{OB}$ ) as well as the presence of spatial clusters.

- This study provided information on the role that place might play in the health status and the prevention of disease, and improving interventions and health-related policies based on spatial heterogeneity.

- The absence of detailed information at the individual level limited research on the determinants of OW/OB on targeted clustered areas.

- As a cross-sectional study, the temporal and spatial trends of $\mathrm{OW} / \mathrm{OB}$ cannot be verified.

overweight or obese. ${ }^{8}$ Overweight and obese children are more likely to stay obese into adulthood and develop non-communicable diseases like diabetes and cardiovascular diseases at a younger age. ${ }^{9-11}$ Even worse, childhood obesity is associated with a higher chance of premature death and disability in adulthood. $^{12}$

Given its high prevalence and severe impact on health outcomes, researchers had poured attention into the modifiable determinants and driving factors of childhood $\mathrm{OW} / \mathrm{OB}$. Several studies have found that childhood $\mathrm{OW} / \mathrm{OB}$ is multifactorial and complex and is considered to result when various genetic, behavioural, environmental, physiological, social and cultural factors interact. ${ }^{13-16}$ It is increasingly recognised that where we live has an impact on our health. For example, the term 'built environment' mainly determined leisure time physical activity through the availability of parks, playgrounds or sports clubs, road traffic and transportation systems and influenced dietary behaviour by spatial accessibility to food through the patterns of food outlets and restaurants. ${ }^{17}$ Changes in the 


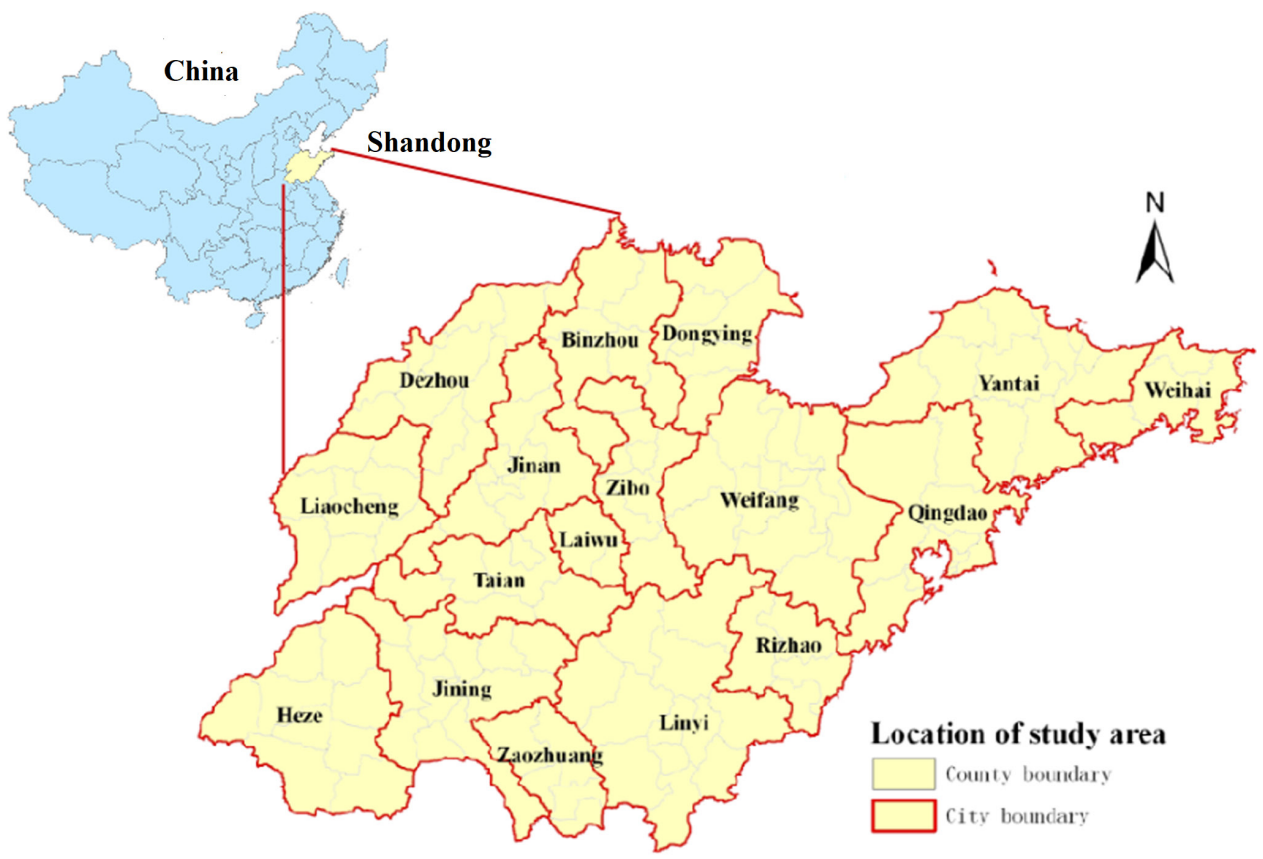

Figure 1 Location of the study area in Shandong Province, China. (The map has obtained permission from the National Administration of Surveying, Mapping and Geoinformation of China.)

lived-in and the built environment have been connected to the rise of obesity. ${ }^{18} 19$ However, the geographical relevance between adjacent regions, especially in terms of lifestyle and social factors, were often neglected in current studies in Chinese schoolchildren. Considering the fact that the prevalence of $\mathrm{OW} / \mathrm{OB}$ at one health region are likely to be correlated with prevalence in nearby regions, ignoring the clustering effect could potentially lead to biassed estimation. ${ }^{20}$

Geographic Information System (GIS) is a potentially 'powerful evidence-based practice tool' in community health, as it enables the identification of spatial epidemiology or the incidence of disease and can allow for effective allocation of resource in a spatial setting. ${ }^{21}$ GIS has been employed in several innovative studies of OW/OB. For example, to explore regional variations and spatial clustering of obesity rates and to identify locations for targeted obesity intervention efforts. ${ }^{18}{ }^{182-24}$ In addition, most of the previous analysis was conducted using large geographical units such as provincial or city level, making it difficult to provide effective implementation of local policies or decisions for obesity prevention. Selecting the most appropriate scale or the smaller geographical areas (ie, counties) makes the obesity interventions more targeted.

Shandong Province is an important littoral province in East China with a population of 99.46 million in 2016 and covers an area of $156700 \mathrm{~km}^{2}$. It includes 140 counties belonging to 17 administration regions (figure 1). The prevalence of childhood obesity in Shandong Province in 2010 reached $15.83 \%$ and $7.12 \%$ among boys and girls, respectively. ${ }^{25}$ In addition, the report of Primary and Secondary Schoolchildren Physical Examination in Shandong province in 2015 showed that the overall overweight and obesity rates of children and adolescent were $30.8 \%$ and $12.8 \%$, respectively. ${ }^{26}$ The childhood OW/OB rates in Shandong province grew rapidly which has caught up the prevalence of childhood OW/OB in developed countries. ${ }^{27}$

Hence, the present study aimed to estimate rates of childhood OW/OB at the county level and apply geographic techniques to examine the spatial patterns of childhood OW/OB by gender, in particular to identify spatial cluster of areas with higher rates of childhood OW/OB.

\section{METHODS}

\section{Data sources and study design}

This study comprised a cross-sectional secondary data analysis of the spatial distribution of childhood OW/OB, using data from Primary and Secondary Schoolchildren Physical Examination Database of Shandong Province. This database contains schoolchildren aged 7-18 years old from all public primary and secondary schools and vocational schools in 2017.

Shandong Province began to conduct health checkups for primary and secondary schoolchildren in 2009 according to the requirements of the National Health Commission of China and apply free health check-up for them every year. Primary and Secondary Schoolchildren Physical Examination is organised by the Health Commission of Shandong Province (the author Wang Lu is one of the leaders of the investigation team). The contents of the medical examination mainly include medicine and surgery, ophthalmology, stomatology, physical indicators and physiological function indicators. The specific process is as follows. Before the physical examination, the 
Health Commission of Shandong Province selected and published the declared medical and health institutions. According to the published list of medical institutions, educational institutions select the nearest institution to enter schools for health examination of students. All measurements were performed by well-trained health professionals in each medical institution using the same type of apparatus and followed the same procedures and the uniform date. During the period of physical examination, a special supervision team went to each physical examination site to conduct quality inspections. After the physical examination, the medical institutions responsible for the physical examination directly input the physical examination data into the Primary and Secondary Schoolchildren Physical Examination Database of Shandong Province. After the data entry, the expert working group will conduct a random sampling check-up of all the entered physical examination data to ensure the data quality.

The physical examination was conducted by medical staffs from 1373 medical institutions in the whole province. The physical examination rate was $93.96 \%$, and data reporting rate was $100 \%$. All schoolchildren voluntarily joined this study, and both schoolchildren and their parents/guardians were provided written informed consent. We extracted individual information of schoolchildren and adolescent, including codes of administrative divisions, age, gender and measure of height and weight. Body mass index (BMI) was calculated (weight in kilograms divided by height in metres squared: $\mathrm{kg}$ / $\mathrm{m}^{2}$ ). Childhood OW/OB was defined according to the classification reference in different age and gender of the Working Group for Obesity in China, the cut-off points of which are 85th and 95th percentiles of BMI, respectively (overweight: 85 th percentile $\leq \mathrm{BMI}<95$ th percentile; obesity: $\mathrm{BMI} \geq 95$ th percentile).$^{28}$ Using demographic data from the 2010 census as the standard population, we estimated age-standardised rates of OW/OB for schoolchildren and adolescents aged 7-18 years older at the county level. The age-standardised rate is a value that represents the ratio of observed incidence of disease to the expected incidence of disease, whereas a value of zero indicates an area with no observed overweight and obese cases.

This study included 140 counties (city districts, county-level cities and counties, often referred to here as 'counties') of Shandong Province as spatial units. In practice, county is a basic unit of the administrative region in China and is also the registered permanent residence of the schoolchildren, which implements the national policies towards population health and health promotion directly. In addition, China's unique household registration system and student roll management system stipulates that primary school and secondary school must be completed in the household registration area, that is, in the county. Primary and junior middle school admission is according to the admission dicing system based on the proximity principle. There is no big difference between the school districts and residence districts. Although few high school students need to go to school far away from their residential areas, these students are difficult to distinguish. This study included 'county' as spatial units, both the school district and the residential area are in one county. This greatly avoids the difference in childhood obesity rates due to differences between school districts and residential areas.

\section{Statistical analysis}

A two-part analysis was conducted: first, the spatial distribution of age-standardised $\mathrm{OW} / \mathrm{OB}$ rates was mapped for boys and girls. Following this, the second part focused on two aspects of the spatial clustering: the overall 'global' spatial clustering in the prevalence of $\mathrm{OW} / \mathrm{OB}$ and the 'local' patterns of OW/OB.

Global Moran's I statistic is a measure of global spatial autocorrelation, a value representing the similarity in $\mathrm{OW} / \mathrm{OB}$ rates between neighbouring regions, and the mathematical definition of Moran's I is as follows. ${ }^{29} 30$

$$
\text { Moran's } \mathrm{I}=\frac{N \sum_{i=1}^{N} \sum_{j=1}^{N} W_{i j}\left(x_{i}-\bar{x}\right)\left(x_{j}-\bar{x}\right)}{\left(\sum_{i=1}^{N} \sum_{j=1}^{N} W_{i j}\right) \sum_{i=1}^{N}\left(x_{i}-\bar{x}\right)^{2}} \quad(i \neq j)
$$

Where $W_{i j}$ is the spatial weights assigned to pairs of units (ie, counties) between observations $i$ and $j, N$ is equal to the total number of pixels.

The Moran's $I$ statistic ranges from +1 (for positive spatial autocorrelation where high values are proximal to other high values or low values adjacent to low values, ie, clustering) to -1 (for negative autocorrelation where high values tend to be near low values, ie, dispersion). A statistically significant $(Z$ score $\geq 1.96$, $p$ value $<0.05$ ) estimate of Moran's $I$ indicates that neighbouring counties have a similar prevalence rate of $\mathrm{OW} / \mathrm{OB}$, and the cases are likely to cluster at the county level. Non-significant values indicate no spatial autocorrelation or that the data are randomly distributed within the study's geographical boundaries. In the present analysis, neighbours were defined using rooks case adjacency, which considers that all regions with common borders are neighbours.

Moran's I statistic is a global test that does not identify where the clusters are located or what type of spatial autocorrelation is occurring (eg, high-high cluster or lowlow cluster and so on). To evaluate the existence of local clusters, it is necessary to use local statistics. The local indicator of spatial autocorrelation (LISA) was therefore applied as an indicator of local spatial association. ${ }^{20}$ The local spatial autocorrelation decomposes the global measurement into the contributions for each geographic region, detecting homogeneity or heterogeneity in values around a given observation or used as diagnostics for outliers in global patterns. The LISA measures whether, for each health region, the standardised rate of $\mathrm{OW} / \mathrm{OB}$ is closer to the values of its neighbours. Inverse distance weighting squared is again used to conceptualise the spatial relationships. To ensure excellent statistical power and consider the computation times, 999 Monte Carlo 
replications were set, and clusters with statistical significance of $\mathrm{p}<0.05$ were reported.

Cluster maps were created using the results of statistical testing to illustrate the local spatial patterns of significant positive and significant negative local spatial autocorrelation. The local spatial autocorrelation analysis results in five categories of health regions: (1) 'high-high' indicates clustering of high values of age-standardised OW/ OB rates (positive LISA value), (2) 'low-high' indicates that low values are surrounded by high values of age-standardised OW/OB rates (negative LISA value), (3) 'lowlow' indicates clustering of low values of age-standardised OW/OB rates (positive LISA value), (4) 'high-low' indicates that high values are surrounded by low values of age-standardised OW/OB rates (negative LISA value) and (5) 'not significant' indicates that there is no spatial autocorrelation. All analyses were conducted using GeoDA ${ }^{31}$ and ArcGIS V.10.3. The geography files were provided by the National Administration of Surveying, Mapping, and Geo-information of China, the administrative organisation in charge of surveying and mapping undertaking across China.

\section{Patient and public involvement}

No patients or public were involved in this study.

\section{RESULTS}

\section{Participants}

After data cleaning, 6216076 qualified schoolchildren aged 7-18 years were finally included for analysis. Participating children were primarily Han ethnicity $(>80 \%)$ and were approximately equally distributed across sex $(50.57 \%$ girls) with a mean (SD) age of 10.94 (2.62) years.

\section{Prevalence of overweight and obesity}

In Shandong province, the overall age-standardised rate of OW/OB was $15.05 \%$ and $9.23 \%$, respectively, which were higher than the national average rates of $5.45 \%$ and $2.83 \%$. Also, the rate of overweight was $17.40 \%$ for boys and $12.73 \%$ for girls; the rate of obesity was $10.49 \%$ for boys and $7.97 \%$ for girls. The age-standardised rate of
$\mathrm{OW} / \mathrm{OB}$ in the county level was higher in boys than girls $\left(\chi^{2}=30.44, \mathrm{p}<0.001\right)$.

Age-standardised rates of overweight differed substantially across health regions ranging from 9.3\% in Qihe, Dezhou to $24.94 \%$ in Penglai, Yantai for boys and from $8.19 \%$ in Linshu, Linyi to $19.15 \%$ in Longkou, Yantai for girls. Obesity rates varied substantially across health regions: from $3.59 \%$ in Changle, Weifang to $20.26 \%$ in Central District in Jining for boys and $3.45 \%$ in Zhanhua, Liaocheng to $13.5 \%$ in Central District in Jining for girls. Age-standardised rates were also mapped by gender at the county level and then classified by the rates (figures 2 and $3)$.

\section{Spatial autocorrelation and cluster identification}

Based on the aforementioned, there was a marked geographical variation of children overweight/obesity in different regions indicating potential presence of spatial clusters or spatial heterogeneity. The Moran's $I$ statistic was applied to test the overall spatial clustering. The results showed that there are significant positive spatial autocorrelations in the data. In particular, the Moran's $I$ statistic of age-standardised overweight rates was 0.3736 (p value $=0.001$ ) for boys and 0.3370 for girls ( $\mathrm{p}$ value $=0.001$ ) . In addition, the Moran's $I$ statistic of age-standardised obesity rates was 0.2312 ( $p$ value $=0.001$ ) for boys and 0.1575 ( $\mathrm{p}$ value $=0.006$ ) for girls. The level of spatial autocorrelation across health regions for age-standardised $\mathrm{OW} / \mathrm{OB}$ rates were slightly higher in boys than girls. For both sexes, the Moran's $I$ statistic and corresponding $\mathrm{p}$ values all suggest non-randomness in the overall spatial pattern of the age-standardised rates of overweight and obesity in Shandong province. Table 1 illustrates spatial correlation statistics and corresponding $\mathrm{p}$ values as estimated by the Moran's I statistic.

We applied LISA to explore more information on the type and location of clustering. County-level LISA significant clusters were observed for both OW/OB (figures 4 and 5). For overweight boys, two larger hot spots (highhigh $(\mathrm{HH})$ ) was detected in the most counties of Yantai in eastern coastal region and Jining in the southwest,
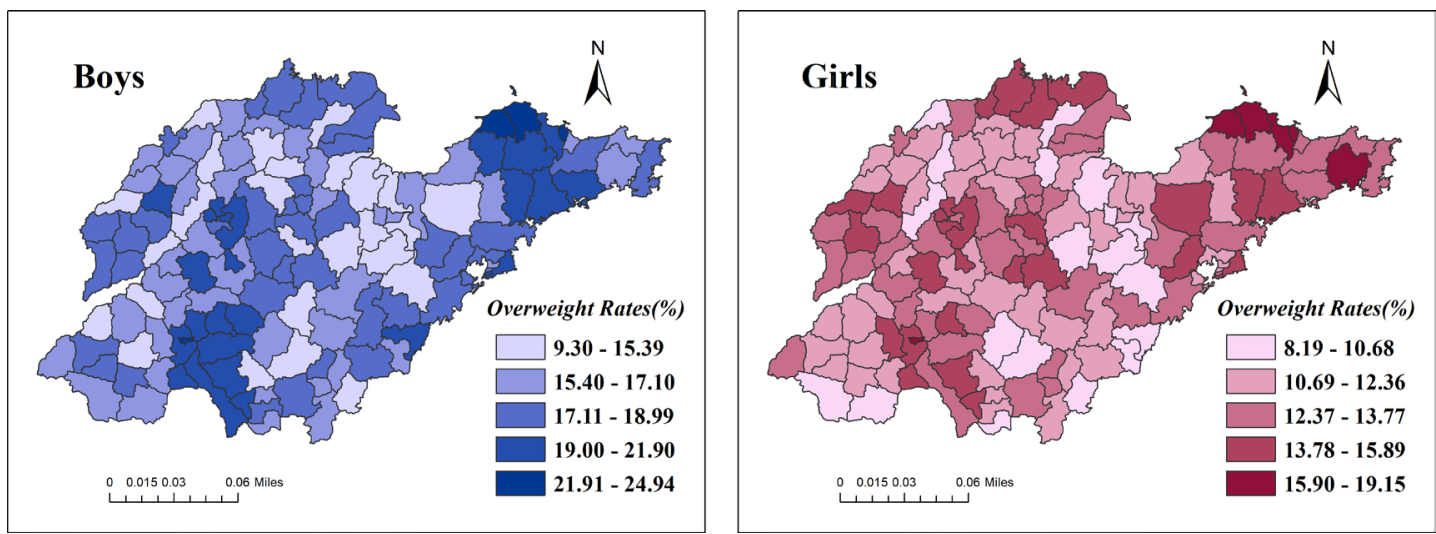

Figure 2 Age-standardised overweight rates for boys and girls by county level in Shandong province. (The map has obtained permission from the National Administration of Surveying, Mapping and Geoinformation of China.) 

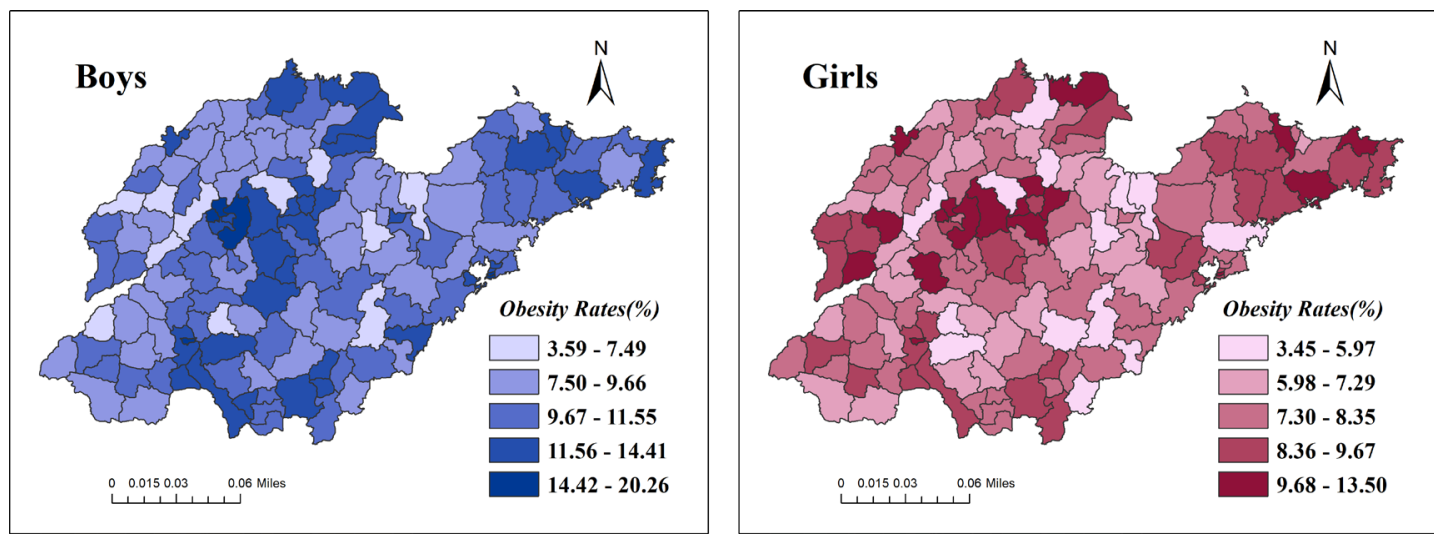

Figure 3 Age-standardised obesity rates for boys and girls by county level in Shandong province. (The map has obtained permission from the National Administration of Surveying, Mapping and Geoinformation of China.)

and one smaller hot spot $(\mathrm{HH})$ was found in Jinan in the central province. Two large cool spots (low-low (LL)) were observed in the eastern and northwestern regions of Shandong province, and one smaller LL was found in a county in southwest. For the girls, the hot spot was comprised of the most counties in Yantai and two districts in Jinan and two counties in Jining. Two large cool spots were detected in the eastern regions (figure 4). However, the pattern of clusters for obesity was different compared with that of overweight (figure 5). For boys, the hot spot mainly distributed in the central region, and a small hot spot was detected in Qingdao in the eastern coastal region. For girls, three hot spots were detected in Weihai in the eastern coastal region, and in Jinan and Zibo in the central region and Jining in the southwest. Also, several counties showed a dispersed pattern of the cluster (highlow (HL) or low-high (LH)) as displayed in figures 4 and 5 .

\section{DISCUSSIONS}

The present study illustrates the marked variability of OW/OB prevalence in Shandong province at the gender and health region level. The results also indicated that the age-standardised rate of both overweight and obesity among boys was higher compared with girls. Our spatial

\begin{tabular}{|c|c|c|c|}
\hline & Moran's I & $Z$ value & $P$ value \\
\hline \multicolumn{4}{|l|}{ Overweight } \\
\hline Boys & 0.3736 & 7.1726 & $0.001^{* *}$ \\
\hline Girls & 0.3370 & 6.1104 & $0.001^{* *}$ \\
\hline Both sexes & 0.2704 & 5.1601 & $0.001^{* *}$ \\
\hline \multicolumn{4}{|l|}{ Obesity } \\
\hline Boys & 0.2312 & 4.4350 & $0.001^{* *}$ \\
\hline Girls & 0.1575 & 3.0727 & $0.006^{* *}$ \\
\hline Both sexes & 0.1130 & 2.2082 & $0.014^{*}$ \\
\hline
\end{tabular}

${ }^{\star} \mathrm{P}<0.05 ;{ }^{\star \star} \mathrm{P}<0.01$. analysis showed the presence of spatial heterogeneity using a significant positive spatial autocorrelation identified by Moran's $I$ statistic for both boys and girls as well as the significant local clusters identified by LISA across the province.

The first concern is the Primary and Secondary Schoolchildren Physical Examination Database of Shandong Province. The census of physical examination for primary and secondary schoolchildren began in Shandong Province in 2009 and examined the physical condition of primary and secondary schoolchildren every year. With a census dataset, the physical health of every student can be mastered in addition to provide evidence for understanding the overall health of students. Several types of research have explored childhood health with the data from the National Surveys on Chinese Students' Constitution and Health in Shandong Province. The survey is conducted every 5 years, and the data are obtained by sampling method, and the existence of sampling errors makes the data less stable. Although the census design increases the accuracy and stability of the data, the absence of other information at the individual level limited research on the factors of schoolchildren's health, such as socioeconomic factors, parental background and psychological status.

The difference in OW/OB between boys and girls is consistent with results from previous studies in Shandong and throughout China. ${ }^{32}{ }^{33}$ Differences in age structure cannot explain this finding, as data have been age standardised. Instead, in addition to the possible biological/ genetic factors, behavioural factors, family environment and psychological factors (eg, body image perceptions) are also related to $\mathrm{OW} / \mathrm{OB}$ in children. On a behavioural level, girls report being more attentive to food and its effects on health and weight control. ${ }^{34}$ A study reported that consumption of foods and beverages outside three main meals and potato chips were more popular in boys than in girls. ${ }^{35}$ Moreover, a review of studies found that boys are also higher users of TV and video games than girls, and these sedentary behaviours have also been associated with body composition and BMI. ${ }^{36}$ There is some evidence that misconceptions about a child's weight 

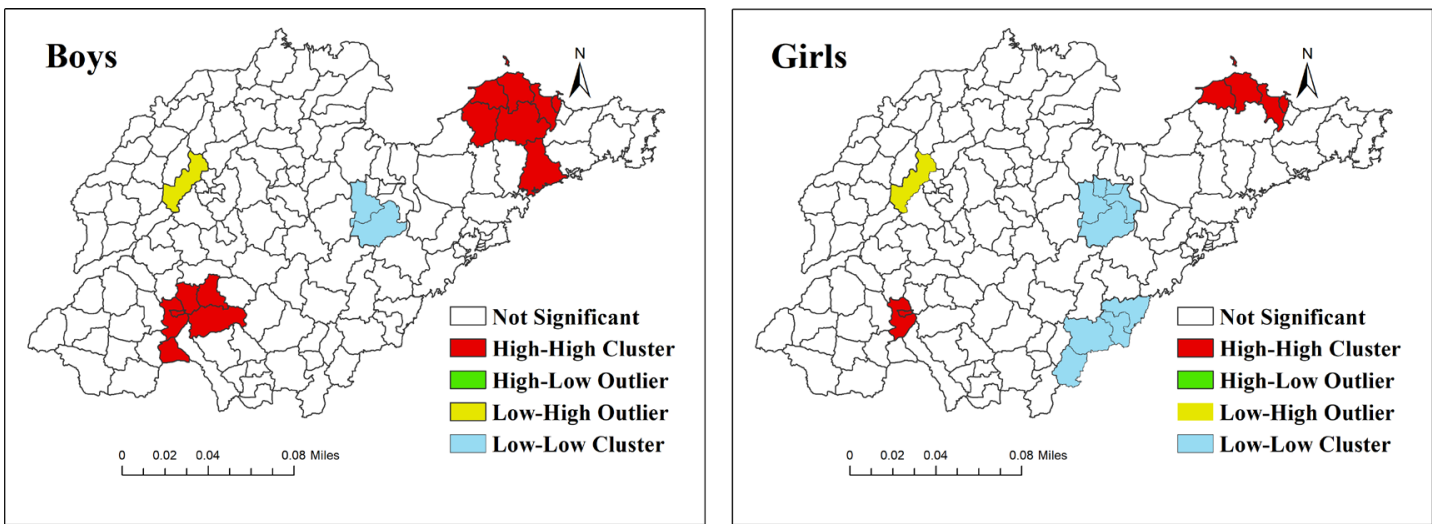

Figure 4 Local indicator of spatial autocorrelationcluster maps for age-standardised overweight rates by county level in Shandong Province. (The map has obtained permission from the National Administration of Surveying, Mapping and Geoinformation of China.)

status were prevalent among parents and grandparents, and boys' weight status was more frequently underestimated than girls. ${ }^{37}$ On a psychological level related to body image, girls self-perceive as overweight more often than do boys. ${ }^{38}$

The global spatial autocorrelation demonstrates that there is significant positive spatial autocorrelation in the data, which showed higher levels of correlation in similar $\mathrm{OW} / \mathrm{OB}$ rates between neighbouring counties in the whole province. The spatial patterns of $\mathrm{OW} / \mathrm{OB}$ might be explained by some possible reasons for neighbourhood characteristics. First, neighbouring county residents may share similar socioeconomic backgrounds; a well-established correlate to obesity. ${ }^{39}$ Second, as residents in neighbouring areas are likely to share similar lifestyles about diet, nutritional habits and physical activity, it could be that residents of adjacent areas with similar obesity rates, share behaviours that promote this condition. ${ }^{18}$ Moreover, adjacent areas also share similar social and built characteristics and therefore the clustered areas may also share similar features. It is worth noting that racial/ethnic differences might also be a possible reason. A previous study has shown that living in neighbourhoods with a higher proportion of foreign-born residents is associated with reduced child obesity risk. ${ }^{40}$ Further research would be necessary to tease out the actual mechanism driving the gradient in $\mathrm{OW} / \mathrm{OB}$ in these areas. In addition, prospective cohort studies and experimental studies were needed to explore the long-term effects of neighbourhood environment directly to the development of obesity.

The LISA found also that the hot spots $(\mathrm{HH})$ were detected in Yantai of the eastern coastal region, Jinan in the central region and Jining in the southwestern inland region among both boys and girls. Again, the cold spots (LL) and other dispersed patterns of the cluster (HL or LH) were observed. Some potential explanations for the different type of clusters are as follows. First, the regional socioeconomic status (SES) might be the influenced factor responsible for the geographic differences in the prevalence of childhood obesity. Some previous studies had confirmed that the regional SES affected to the distribution of $\mathrm{OW} / \mathrm{OB}$ positively in China. In other words, high SES youths are more likely to be OW/OB than other lower SES counterparts. ${ }^{41}$ The evidence provided by research indicated that $\mathrm{OW} / \mathrm{OB}$ prevalence was greater and increased more quickly in the coastal counterparts. ${ }^{7}$ Eastern coastal region has high SES with a more developed economy, the living standards, nutritional conditions and
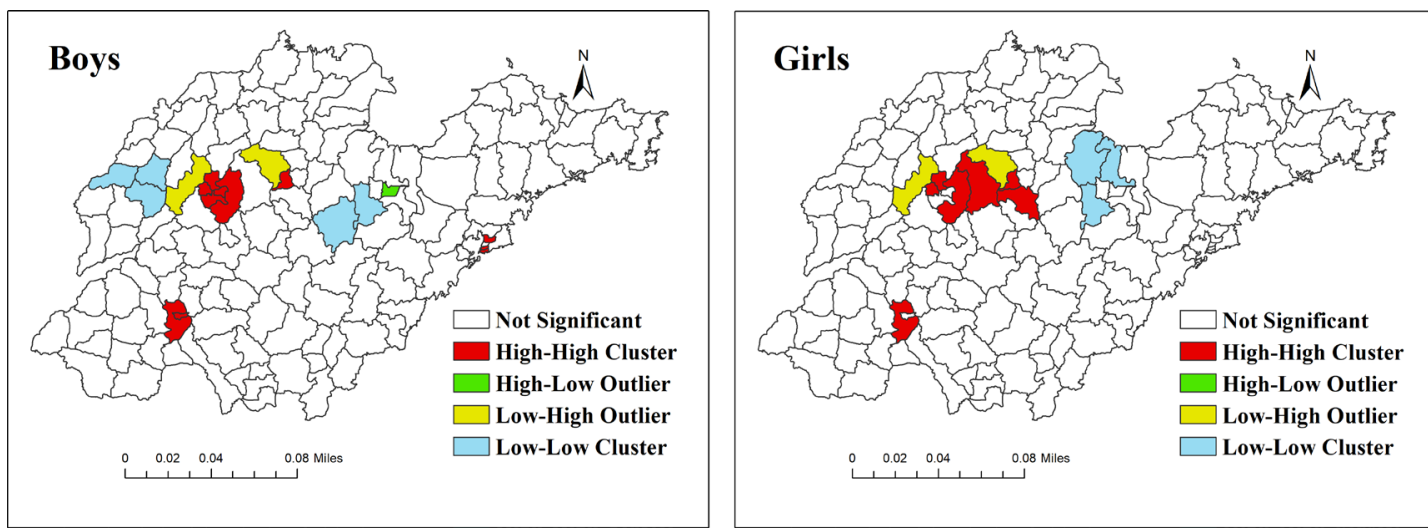

Figure 5 Local indicator of spatial autocorrelation cluster maps for age-standardised obesity rates by county level in Shandong Province. (The map has obtained permission from National Administration of Surveying, Mapping and Geoinformation of China.) 
public health are better than other SES districts. Second, with the fast-growing Chinese economy, the rapid urbanisation has narrowed the disparity between coastal and inland counterparts and became a potential contributor that cannot be ignored. ${ }^{42}{ }^{43}$ That is the reason why the hot spot of OW/OB for children also occurred in the inland region. In addition to the aforementioned factors, the traditional Chinese diet is shifting towards a diet with high fat, high energy density and low dietary fibre on the whole, changes which have resulted in rapid increases in the prevalence of OW/OB ${ }^{44}$ Moreover, television viewing or surfing the Internet besides reducing physical activity also leads to increased consumption of energy-rich foods through incessant commercial advertisement. ${ }^{45}$ These factors lead to a rapid increase in the prevalence of overweight and obesity among children and adolescent.

The strength of this study is that the data come from the Primary and Secondary Schoolchildren Physical Examination Database with a large sample size in Shandong Province, China. Examination survey provided direct measurements of weight and height and avoided bias in self-reported data. The results are important for understanding the role that place might play in the health status and suggesting the importance of improving interventions and health-related policies that are thus far mainly based on assumptions of spatial homogeneity. In addition, the findings verified the effectiveness of spatial data analysis methods and in particular the helpful means of cluster analysis for public health professionals and policymakers. For instance, based on the potential explanations for the identified clusters, existing policies and practices could provide funding for physical education as well as recreation centres in communities most in need. Furthermore, it may be advantageous from the perspective that exploring areas of low prevalence to ascertain which factors might decrease the risk of the population. Further research is required to explore the underlying mechanism for the increased levels of $\mathrm{OW} / \mathrm{OB}$ on clustered areas and investigate the impact of area-level characteristics on the spatial patterns identified.

This study has several limitations. First, the absence of detailed information at the individual level limited research on the determinants of $\mathrm{OW} / \mathrm{OB}$ on targeted clustered areas. Second, the study may also be affected by the modifiable areal unit problem. The spatial scale used is not unique and typically arbitrary and modifiable; the spatial patterns of OW/OB identified may change depending on different scales. Also, we only analysed the data for 1 year, due to data availability, the temporal and spatial trends of OW/OB cannot be verified.

\section{CONCLUSIONS}

A high prevalence of childhood OW/OB was observed in Shandong Province, one of the populous provinces in China. There were marked sex-specific regional differences in the prevalence of childhood OW/OB, along with a tendency of spatial correlation of the childhood
OW/OB. In addition, the spatial clusters provide valuable information on the identification of local geographical variation and elevated risk of $\mathrm{OW} / \mathrm{OB}$ in Shandong province. Therefore, prevention and health promotion interventions, as well as effective allocation of resources and services, should be implemented based on regional and population-specific demands. For the hot spots of childhood OW/OB, resource allocation is mainly concentrated in three aspects. First, through the health education of parents, they are encouraged to establish a healthy lifestyle, which in turn affects children's cognition and diet behaviour. In addition, the schools should develop appropriate health strategies, including education on nutrition knowledge, increased physical activity and encourage children to establish health behaviours. Furthermore, the government should provide more interventions for hot spots of childhood OW/OB, including developing dietary guidelines, increasing community exercise facilities and conducting health education.

Acknowledgements We would like to thank Shandong University Center for Health Economics Experiment and Public Policy Research; NHC Key Laboratory of Health Economics and Policy Research (Shandong University) for the supports.

Contributors WQ planned the study, conducted the analysis and wrote the paper while being supervised by LX; LW, JL, LS helped to plan the study, including the instrumentation and to revise the manuscript. JZ and HS accomplished the statistical analysis and contributed to revising the paper. All authors contributed to the discussion of the paper, read and approved the final manuscript.

Funding This project was funded by the National Natural Science Foundation of China (grant number 71673169 and 71673170 ).

Map disclaimer The depiction of boundaries on the map(s) in this article do not imply the expression of any opinion whatsoever on the part of BMJ (or any member of its group) concerning the legal status of any country, territory, jurisdiction or area or of its authorities. The map(s) are provided without any warranty of any kind, either express or implied.

\section{Competing interests None declared.}

Patient consent for publication Not required.

Ethics approval This study was approved by the Academic Research Ethics Committee of Shandong University.

Provenance and peer review Not commissioned; externally peer reviewed. Data availability statement № data are available.

Open access This is an open access article distributed in accordance with the Creative Commons Attribution Non Commercial (CC BY-NC 4.0) license, which permits others to distribute, remix, adapt, build upon this work non-commercially, and license their derivative works on different terms, provided the original work is properly cited, appropriate credit is given, any changes made indicated, and the use is non-commercial. See: http://creativecommons.org/licenses/by-nc/4.0/.

\section{REFERENCES}

1. WHO. Childhood overweight and obesity, 2018. Available: http:// www.who.int/dietphysicalactivity/childhood/en/

2. Ogden CL, Carroll MD, Kit BK, et al. Prevalence of childhood and adult obesity in the United States, 2011-2012. JAMA 2014;311.

3. Stamatakis E, Wardle J, Cole TJ. Childhood obesity and overweight prevalence trends in England: evidence for growing socioeconomic disparities. Int J Obes 2010;34:41-7.

4. Abarca-Gómez L, Abdeen ZA, Hamid ZA, et al. Worldwide trends in body-mass index, underweight, overweight, and obesity from 1975 to 2016: a pooled analysis of 2416 population-based measurement studies in 128.9 million children, adolescents, and adults. Lancet 2017;390:2627-42.

5. Cheng TO. Obesity in Chinese children. J R Soc Med 2004;97:254. 
6. CY J. The prevalence of childhood overweight/obesity and the epidemic changes in 1985-2000 for Chinese school-age children and adolescents. Obes Rev 2010;9:78-81.

7. Ji CY, Cheng TO. Epidemic increase in overweight and obesity in Chinese children from 1985 to 2005. Int J Cardiol 2009;132:1-10.

8. $\mathrm{Ng} \mathrm{M}$, Fleming $\mathrm{T}$, Robinson $\mathrm{M}$, et al. Global, regional, and national prevalence of overweight and obesity in children and adults during 1980-2013: a systematic analysis for the global burden of disease study 2013. Lancet 2014;384:766-81.

9. Wang Y, Lobstein T. Worldwide trends in childhood overweight and obesity. Int J Pediatr Obes 2006;1:11-25.

10. Kelly AS, Barlow SE, Rao G, et al. Severe obesity in children and adolescents: identification, associated health risks, and treatment approaches: a scientific statement from the American heart association. Circulation 2013;128:1689-712.

11. Bridger T. Childhood obesity and cardiovascular disease. Paediatr Child Health 2009;14:177-82.

12. Peeters A, Barendregt JJ, Willekens F, et al. Obesity in adulthood and its consequences for life expectancy: a life-table analysis. Ann Intern Med 2003;138:24-32.

13. Shan $\mathrm{X}-\mathrm{Y}, \mathrm{Xi} \mathrm{B}$, Cheng $\mathrm{H}$, et al. Prevalence and behavioral risk factors of overweight and obesity among children aged 2-18 in Beijing, China. Int J Pediatr Obes 2010;5:383-9.

14. Lin M, Pan L, Tang L, et al. Association of eating speed and energy intake of main meals with overweight in Chinese pre-school children. Public Health Nutr 2014;17:2029-36.

15. Song Y, Zhang X, Ma J, et al. Behavioral risk factors for overweight and obesity among Chinese primary and middle school students in 2010. Zhonghua yu fang yi xue za zhi 2012;46. Article in Chinese.

16. Fang $D$, Thomsen MR, Nayga RM, et al. Association of neighborhood geographic spatial factors with rates of childhood obesity. JAMA Netw Open 2018;1.

17. Story M, Kaphingst KM, Robinson-O'Brien R, et al. Creating healthy food and eating environments: policy and environmental approaches. Annu Rev Public Health 2008;29:253-72.

18. Paisi M. Obesity and dental caries in children in plymouth: University of plymouth, 2017. Available: http://hdl.handle.net/10026.1/9582

19. Casey R, Oppert J-M, Weber C, et al. Determinants of childhood obesity: what can we learn from built environment studies? Food Qual Prefer 2014;31:164-72.

20. Anselin L. Local indicators of spatial Association-LISA. Geogr Anal 1995;27:93-115.

21. McLafferty SL. GIS and health care. Annu Rev Public Health 2003;24:25-42.

22. Fraser LK, Clarke GP, Cade JE, et al. Fast food and obesity: a spatial analysis in a large United Kingdom population of children aged 13-15. Am J Prev med 2012;42:e77-85.

23. Pouliou T, Elliott SJ. An exploratory spatial analysis of overweight and obesity in Canada. Prev Med 2009;48:362-7.

24. Hughey SM, Kaczynski AT, Porter DE, et al. Spatial clustering patterns of child weight status in a southeastern us County. Appl Geogr 2018;99:12-21.

25. Zhang $Y X$, Wang SR. Secular trends in body mass index and the prevalence of overweight and obesity among children and adolescents in Shandong, China, from 1985 to 2010. J Public Health 2011:34:131-7.

26. Qi Y, Li N, Jia T, et al. Analysis on the relevant factors of obesity complicated with hypertension of overweight students from "health summer camp" of elementary and secondary schools in Shandong Province in 2015. China Medical Herald 2016;13:89-92. Article in Chinese.

27. Wang $Y$, Lim H. The global childhood obesity epidemic and the association between socio-economic status and childhood obesity. Int Rev Psychiatry 2012;24:176-88.

28. Group of China Obesity Task Force. Body mass index reference norm for screening overweight and obesity in Chinese children and adolescents. Zhonghua liu xing bing xue za zhi 2004;25:97. Article in Chinese.

29. Pfeiffer D, Robinson TP, Stevenson M, et al. Spatial analysis in epidemiology. Oxford University Press Oxford, 2008.

30. Wheeler D, Shaw G, Barr S. Statistical techniques in geographical analysis. Routledge, 2013.

31. Anselin L, Syabri I, Kho Y. GeoDa: an introduction to spatial data analysis. Geogr Anal 2006;38:5-22.

32. Zhang T, Cai L, Ma L, et al. The prevalence of obesity and influence of early life and behavioral factors on obesity in Chinese children in Guangzhou. BMC Public Health 2016;16:954.

33. Guo X, Zheng L, Li Y, et al. Prevalence and risk factors of being overweight or obese among children and adolescents in northeast China. Pediatr Res 2013;74:443-9.

34. Wardle J, Haase AM, Steptoe A, et al. Gender differences in food choice: the contribution of health beliefs and dieting. ann. behav. med. 2004;27:107-16.

35. Li M, Dibley MJ, Sibbritt DW, et al. Dietary habits and overweight/ obesity in adolescents in Xi'an City, China. Asia Pac J Clin Nutr 2010;19:76-82.

36. Marshall SJ, Gorely T, Biddle SJH. A descriptive epidemiology of screen-based media use in youth: a review and critique. J Adolesc 2006;29:333-49.

37. Li J, Lei J, Wen S, et al. Sex disparity and perception of obesity/ overweight by parents and grandparents. Paediatr Child Health 2014;19:e113-6.

38. O'Dea JA. Gender, ethnicity, culture and social class influences on childhood obesity among Australian schoolchildren: implications for treatment, prevention and community education. Health Soc Care Community 2008;16:282-90.

39. Penney TL, Rainham DGC, Dummer TJB, et al. A spatial analysis of community level overweight and obesity. J Hum Nutr Diet 2014;27:65-74.

40. Kimbro RT, Denney JT. Neighborhood context and racial/ethnic differences in young children's obesity: structural barriers to interventions. Soc Sci Med 2013;95:97-105.

41. Zhang Y-X, Wang S-R. Differences in development and the prevalence of obesity among children and adolescents in different socioeconomic status districts in Shandong, China. Ann Hum Biol 2012;39:290-6.

42. Contaldo F, Mazzarella G, Santarpia L, et al. Influence of urbanization on childhood obesity. Nutr Metab Cardiovas 2015;25:615-6

43. Zhang Y-xiu, Zhao J-shan, Chu Z-hua. Prevalence of overweight and obesity among children and adolescents is associated with urbanization in Shandong, China. Int J Cardiol 2014;176:1212-3.

44. Du S, Lü B, Wang Z, et al. Transition of dietary pattern in China. Journal of hygiene research 2001;30:221-5. Article in Chinese.

45. Matheson DM, Killen JD, Wang Y, et al. Children's food consumption during television viewing. Am J Clin Nutr 2004;79:1088-94. 